\title{
Receptor-Activated SMAD
}

National Cancer Institute

\section{Source}

National Cancer Institute. Receptor-Activated SMAD. NCI Thesaurus. Code C113248.

A family of transcription factors that are activated by signaling mediated by tumor growth factor-beta (T GF-beta) superfamily ligand-receptor interactions. Once activated, R-SMADs play a role in the positive regulation of T GF-beta responsive gene expression. 